\title{
UV laser ablation of parylene films from gold substrates
}

\author{
O. R. Musaev, P. Scott, J. M. Wrobel, and M. B. Kruger
}

National Nuclear Security Administration's Kansas City Plant*

Department of Physics, University of Missouri - Kansas City, Kansas City, MO 64110

\begin{abstract}
Parylene films, coating gold substrates, were removed by laser ablation using $248 \mathrm{~nm}$ light from an excimer laser. Each sample was processed by a different number of pulses in one of three different environments: air at atmospheric pressure, nitrogen at atmospheric pressure, and vacuum. The laser-induced craters were analyzed by optical microscopy and x-ray photoelectron spectroscopy. Multi-pulse ablation thresholds of gold and parylene were estimated.
\end{abstract}

${ }^{*}$ Operated for the United States Department of Energy under Contract No. DE-ACO4-01AL66850. 


\section{Introduction}

Parylene is an organic polymer that has a variety of technological applications. For example, it is used as a protective layer in printed circuit boards, as a flexible substrate for microwave integrated circuits, and as a biocompatible coating for microelectrodes in neural science. ${ }^{1-4}$ Selective parylene removal is required for various applications. Chemical removal is problematic due to the inert nature of parylene; plasma etching gives better results. ${ }^{2}$ However, plasma etching is more complex in comparison with laser patterning.

Laser ablation of polymers has attracted attention since the early eighties. ${ }^{5}$ It has become an area of interest in various fields including the microelectronics industry, bioengineering and chemistry. ${ }^{6}$ Laser ablation of parylene was performed as early as 1991 and has more recently been used in developing high aspect ratio three-dimensional multi-electrode MEMS arrays and to remove parylene from the tips of biomedical microelectrodes. ${ }^{3,7-9}$

For fabrication purposes it is often necessary to remove a thin layer of parylene from a metal surface without damaging the substrate. In this work we studied laser ablation of parylene from gold surfaces.

\section{Experimental}

The samples studied were either bare gold, or gold coated with a layer of parylene-C. A Lambda Physics KrF excimer laser, model LPX-210, operating at $248 \mathrm{~nm}$ with pulse duration of $20 \mathrm{~ns}$, was used. Homogenization of the beam was achieved by expansion of the beam and application of a circular aperture. A small fraction $(\sim 1 \%)$ of the radiation coming from the laser was transmitted through the hole in the aperture. A three-element converging lens system focused the light to a $0.5 \mathrm{~mm}$ diameter circle on the sample's surface. The fluence in the processes was varied between $300 \mathrm{~mJ} / \mathrm{cm}^{2}$ to $800 \mathrm{~mJ} / \mathrm{cm}^{2}$. Samples were processed in air at atmospheric pressure, nitrogen at atmospheric pressure, and vacuum.

For determination of the ablation threshold and processing rates of both parylene and gold, depths of the ablation craters were optically measured and microphotographs of the laser processed craters were taken. For cases of significant carbon deposits, the surface was cleaned in distilled water in an ultrasonic bath before measurements were made. Chemical analysis of the processed spots with X-ray photoelectron spectroscopy (XPS) in conjunction with optical micrographs was used to determine the effectiveness of parylene removal. The craters were probed for the presence of gold, carbon and chlorine.

Results and discussion

The effect of $248 \mathrm{~nm}$ laser radiation on an uncoated gold surface was investigated. In Figure 1 the damage to the gold surface for four values of fluence is shown. In each case the surface was exposed to 300 laser pulses and the process was carried out in air. Fluence values used here were just above the multi-pulse ablation threshold of gold. One should recognize that 
the fluences used are much lower than the single-pulse ablation threshold which is about $2 \mathrm{~J} / \mathrm{cm}^{2}{ }^{10}$

Using an optical microscope with a high magnification objective $(100 \times)$ the depth of the craters in the gold surface was measured. The obtained experimental dependence of crater depth for 300 pulses on fluence is plotted in Figure 2. The best linear fit and the extrapolation of the relationship to low fluences yield the ablation threshold for gold under these conditions to be about $255 \mathrm{~mJ} / \mathrm{cm}^{2}$.

In order to investigate the effect of accidental exposure of gold to laser radiation after removal of parylene, the effect of number of pulses at the fluence comparable to that used for ablation of parylene was carried out. As shown in Figure 3, the rate at which gold was ablated was not constant but it varied with the number of pulses. The etched depth is a power function of the number of pulses, with the exponent of 0.5 .

Previously a $\mathrm{KrF}$ excimer laser was used to study ablation of parylene from gold films at fluences in the range $6.7-26.7 \mathrm{~mJ} / \mathrm{cm}^{2}$. ${ }^{9}$ In that research an approximately linear dependence of ablation depth on the number of pulses $(200-1000)$ was observed. In the present work, the ablation of parylene films from gold substrates was performed at fluences between $330 \mathrm{~mJ} / \mathrm{cm}^{2}$ and $550 \mathrm{~mJ} / \mathrm{cm}^{2}$. In Figure 4, the depth of ablation by 10 pulses for different fluences is depicted. From this the ablation threshold at 10 pulses is estimated to be $340 \mathrm{~mJ} / \mathrm{cm}^{2}$. This is in good agreement with an estimation that can be obtained from previously published data. ${ }^{7}$

In Figure 5, the depth of parylene removed, at a fluence of $330 \mathrm{~mJ} / \mathrm{cm}^{2}$, is plotted versus the number of pulses for three environments: nitrogen, air and vacuum. The ablated depth is not directly proportional to the number of pulses. As the process continues, the ablation rate decreases. As for the case of gold, the amount of ablated parylene, as a function of the number of pulses, follows a power law. In both nitrogen and air the rates are comparable, however a significantly higher rate is observed in vacuum. The decrease of the ablation rate can be related to the change of morphology or material modification and chemical composition change of the irradiated area. For example, in the ablation of polyimide the ablation rate strongly decreases with the number of pulses. ${ }^{11}$ This occurs for two reasons: roughness increases with the number of pulses which causes a decrease of effective fluence, and carbonization of the material which alters the ablation conditions. The same effects, increased roughness and carbonization occur during ablation of parylene.

Images of ablated craters in parylene on gold, obtained under different conditions, are presented in Figure 6. Ablation in air and nitrogen resulted in large deposits of carbon in the vicinity of the etched craters. As seen in the top row of the figure, the size of the carbon deposits extends about $0.5 \mathrm{~mm}$ from the edge of the crater. An attempt to remove this carbon by placing the samples in distilled water in an ultrasonic bath was effective as can be seen in Figure 6 b. From Figure 6a it is clear that carbon is not deposited on the sample during ablation in vacuum. This can be explained by uninhibited expansion of the plasma plume into vacuum. Carbon particles and soot move with the flow and are dispersed and deposited further away from the irradiated spot. 
Chemical processes taking place during ablation were investigated using XPS. Craters etched under different conditions were studied. As seen in Figure 7, oxygen 1s, carbon 1s, and chlorine $2 p$ lines have been detected in the unprocessed regions of the samples. Oxygen is not present in any of the parylene compounds. It must be either adsorbed on the surface (more probable) or incorporated in some other chemical bond with the remaining elements. In an etched crater, the $4 \mathrm{f}$ line of gold appears in the XPS spectrum. It is possible that a trace of parylene is observed in the crater, as evidenced by the low intensity $\mathrm{Cl} 2 \mathrm{p}$ line, while due to laser processing more oxygen becomes adsorbed or chemically bound at the bottom of the crater.

Distribution maps of gold XPS spectra have been collected for a few of the etched craters after ultrasonic cleaning. An example of a parylene ablation process for craters etched in air at $330 \mathrm{~mJ} / \mathrm{cm}^{2}$ is presented in Figure 7. The top row consists of the optical micrograph of the investigated craters. The corresponding maps of XPS intensity at the $4 \mathrm{f}$ gold line are shown in the bottom row. The non-uniform distribution of the gold line intensity suggests that the gold surface is not completely clean.

In this work it has been demonstrated that the 10 pulse ablation threshold of parylene is greater than the 300 pulse ablation threshold of gold. Under the studied conditions, the complete removal of parylene may result in some damage to the gold substrate. This implies that adaptive control of the laser fluence may be the method of choice for thin film removal without substrate damage. Realistic values for the number of pulses can be in the range between 10 and a few hundred. During processing the thickness of the thin film decreases and consequently illumination of the substrate rises. That increases the possibility of damage to the substrate. Thus, the goal of adaptive control is to decrease fluence with pulse number, in such a way as to maximize film removal while leaving the substrate undamaged.

\section{Conclusion}

Multi-pulse laser ablation thresholds were established for parylene films on gold, and also for the gold substrates using $248 \mathrm{~nm}, 20 \mathrm{~ns}$ pulse light. Scaling exponents for both materials as functions of the number of pulses were evaluated.

Ablation was performed in air, vacuum and nitrogen gas. For ablation in air and nitrogen, carbon was deposited on the surface, which can be removed in an ultrasonic bath. In contrast, vacuum ablation resulted in carbon free surfaces.

It was determined that the ablation threshold for parylene at 10 pulses is $340 \mathrm{~mJ} / \mathrm{cm}^{2}$, while the ablation threshold of gold at 300 pulses is $255 \mathrm{~mJ} / \mathrm{cm}^{2}$. This means that at fluences required for quick removal of parylene, extended exposure of the gold will damage the substrate. However, if the number of laser pulses after removal of parylene is below 10 the damage to the substrate is minimal. Alternatively, reduction of the laser fluence with parylene removal may be employed.

Acknowledgements 
This work was partially supported by grants from the University of Missouri-Research Board and the UMKC Research Board.

Notice: This manuscript has been authored by Honeywell Federal Manufacturing \& Technologies under Contract No.DE-ACO4-01AL66850 with the U.S. Department of Energy. The United States Government retains and the publisher, by accepting the article for publication, acknowledges that the United States Government retains a nonexclusive, paid-up, irrevocable, world-wide license to publish or reproduce the published form of this manuscript, or allow others to do so, for United States Government purposes. 


\section{References}

1 H. C. Hoch, L. Jelinski, and H. G. Craighead, (Cambridge Univ. Press, New York, NY, 2006).

2 E. Meng and Y.-C. Tai, in 18th IEEE Int. Conf. on MEMS (IEEE, Miami, FL, 2005), p. 568

3 E. M. Schmidt, M. J. Bak, and P.Christensen, J. NeuroSci. Meth. 62, 89 (1995).

4 H. Sharifi, R. R. Lahiji, H.-C. Lin, P. D. Ye, L. P. B. Katehi, and S. Mohammadi, IEEE Transactions on Advanced Packaging 32, 85 (2009).

5 R. Srinivasan and V. Mayne-Banton, Appl. Phys. Lett. 41, 576 (1982).

6 T. Lippert and J. T. Dickinson, Chem. Rev. 103, 453 (2003).

7 M. Esashi, K. Minami, and S. Shoji, in MEMS'91 (IEEE, Nara, Japan, 1991).

8 Y. Choi, S.-O. Choi, R. H. Shafer, and M. G. Allen, in 13th Int. Conf on Solid-State Sensors, Actuators and Microsystems, TRANSDUCERS'05 (IEEE, Atlanta, GA, 2005), Vol. 2, p. 1469.

9 F. Herrault, C.-H. Ji, S. Rajaraman, R. H. Shafer, and M. G. Allen, in Intern. Conf. on SolidState Sensors, Actuators and Microsystems 2007. TRANSDUCERS 2007., Lyon, France, 2007), p. 513

10 A. Rosenfeld and E. E. B. Campbell, Appl. Surf. Sci. 96-98, 439 (1996).

11 E. Arenholz, J. Heitz, M. Wagner, D. Bauerle, H. Hibst, and A. Hagenmeyer, Appl. Surf. Sci. 69, 16 (1993). 


\section{Figure Captions}

Figure 1. Surface of gold after illumination with 300 pulses at fluences indicated below each image. Ablation took place in atmosphere.

Figure 2. Determination of the ablation threshold of gold in air at $248 \mathrm{~nm}$ with 300 (20 ns) pulses.

Figure 3. Depth of gold ablated at $248 \mathrm{~nm}$ versus the number of pulses with $310 \mathrm{~mJ} / \mathrm{cm}^{2}$ fluence.

Figure 4. Determination of the ablation threshold of parylene in air at $248 \mathrm{~nm}$ with 10 (20 ns) pulses.

Figure 5. Depth of parylene ablated at $248 \mathrm{~nm}$ in nitrogen, air and vacuum versus the number of pulses. The laser fluence was $330 \mathrm{~mJ} / \mathrm{cm}^{2}$.

Figure 6. Optical microscope images of holes ablated in $\sim 10 \mu \mathrm{m}$ parylene-C layer on gold: a) before cleaning; b) after 3 minutes in an ultrasonic bath.

Figure 7. Exploratory X-ray photoemission spectra (baseline subtracted) from (a) a parylene film and (b) an etched crater.

Figure 8. Evolution of morphology and chemical composition of an ablation crater etched in a $11 \mu \mathrm{m}$ parylene layer at $330 \mathrm{~mJ} / \mathrm{cm}^{2}$ fluence. 


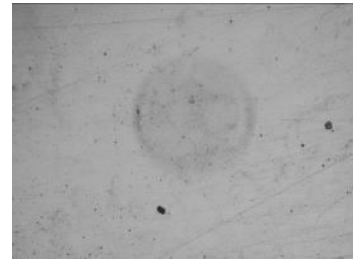

$330 \mathrm{~mJ} / \mathrm{cm}^{2}$

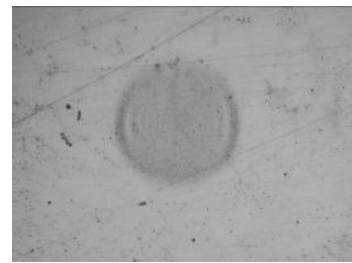

$380 \mathrm{~mJ} / \mathrm{cm}^{2}$

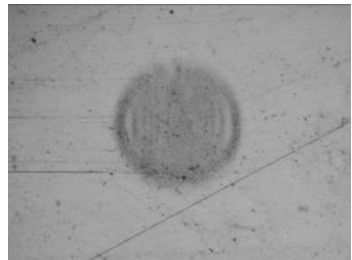

$400 \mathrm{~mJ} / \mathrm{cm}^{2}$

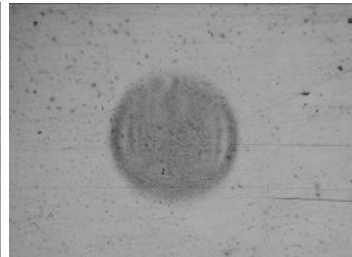

$430 \mathrm{~mJ} / \mathrm{cm}^{2}$

Figure 1. 


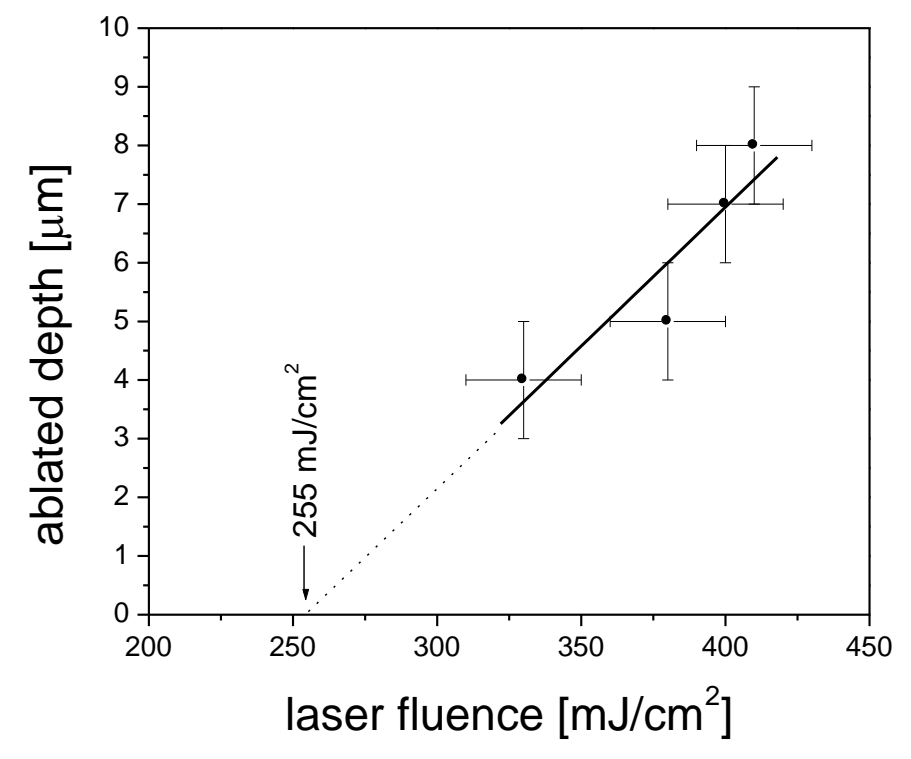

Figure 2. 


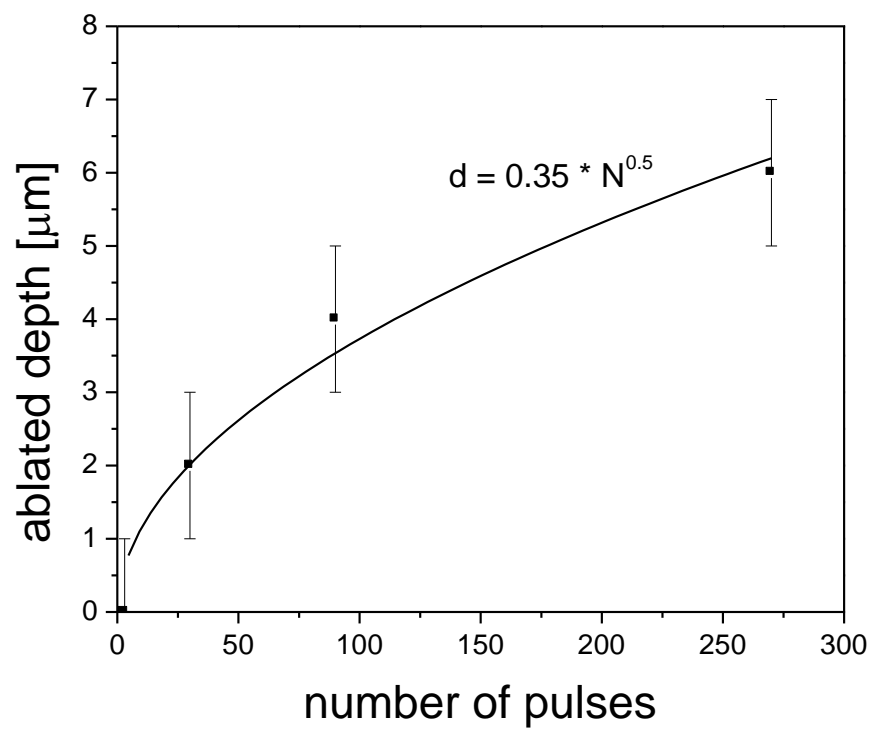

Figure 3. 


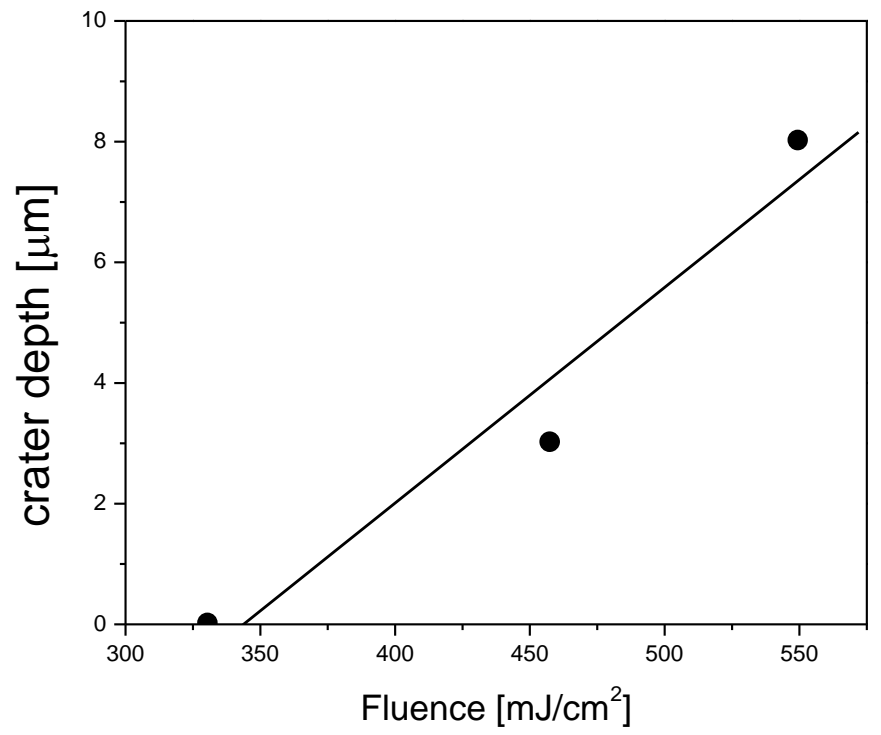

Figure 4 


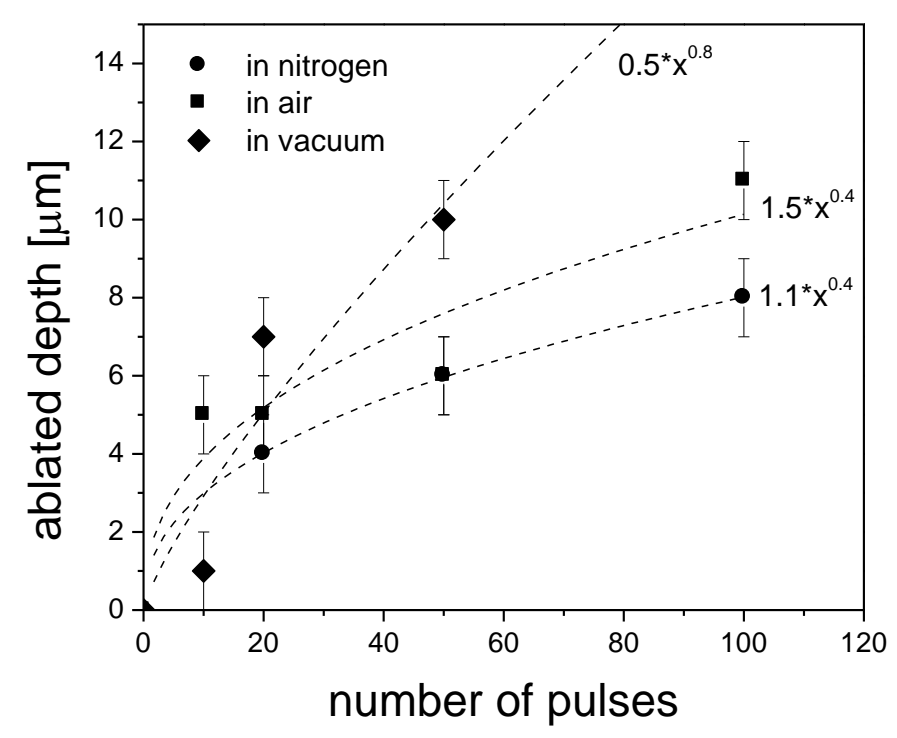

Figure 5. 


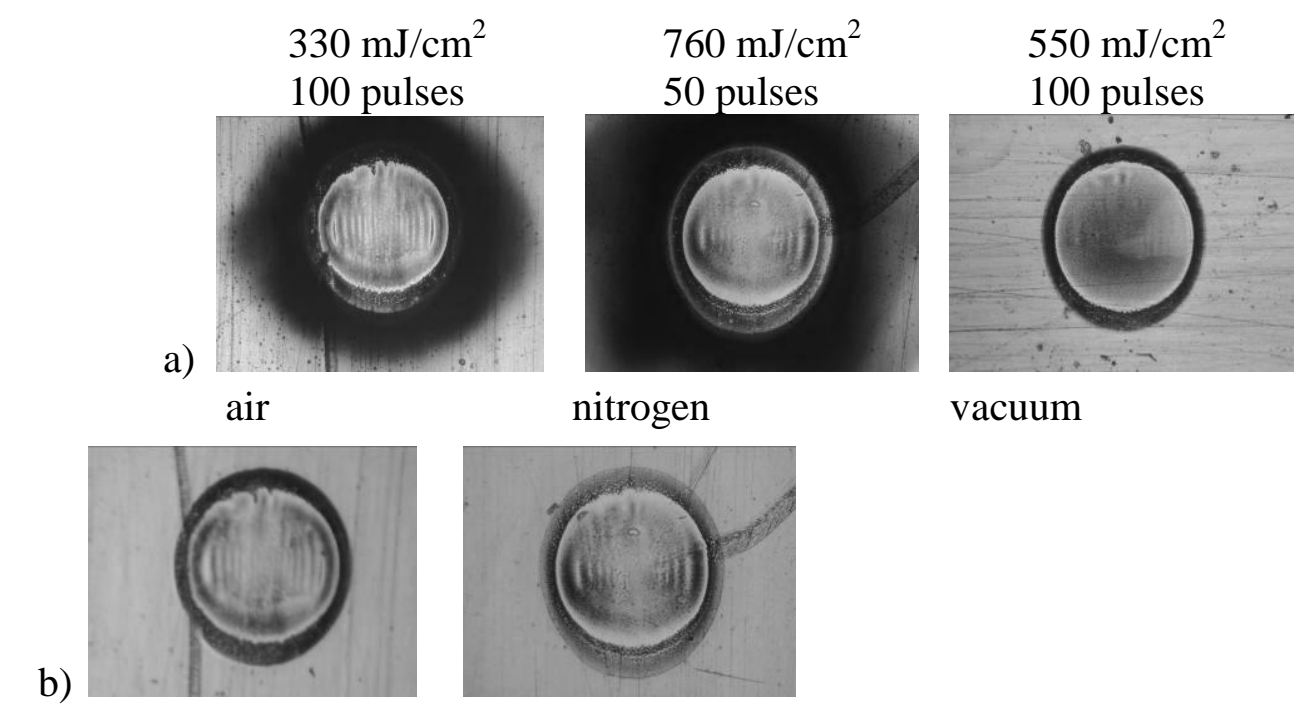

Figure 6. 

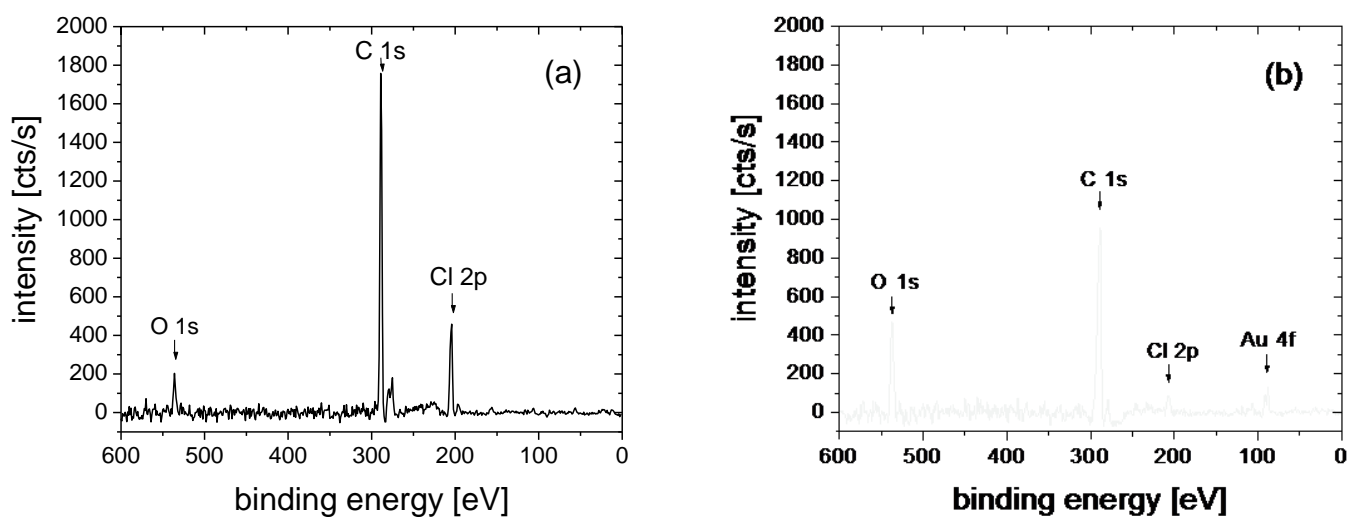

Figure 7. 


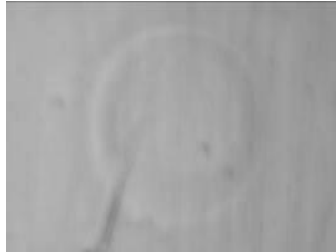

10 pulses

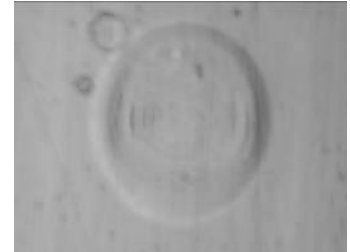

20 pulses

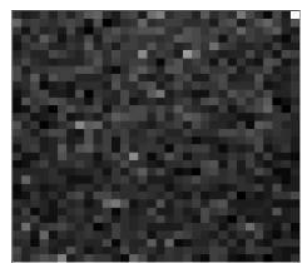

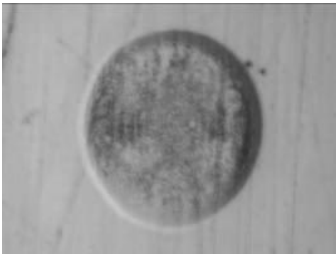

50 pulses

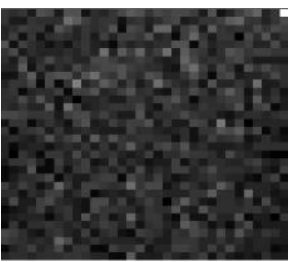

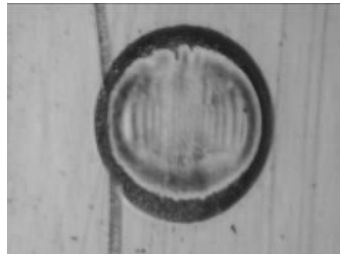

100 pulses

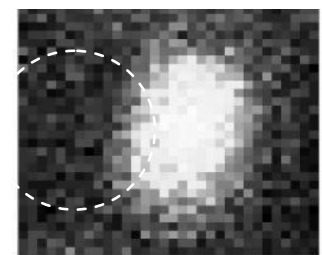

Figure 8. 